\title{
A Tunable Bandstop Resonator Based on a Compact Slotted Ground Structure
}

\author{
Xiao-Hua Wang, Bing-Zhong Wang, Member, IEEE, Hualiang Zhang, and Kevin J. Chen, Senior Member, IEEE
}

\begin{abstract}
A new slotted ground structure featuring compact size and spurious-free passband in the context of microstrip lines is proposed. The compactness of the new structure originates from the lengthened coupling gap in the ground. The spurious-free response in a wide passband is a result of combining both narrow and wide slots in the new structure, which effectively forms a stepped-impedance slot resonator. Compared to the previously proposed dumbbell- and $\mathrm{H}$-shaped defected ground structure, over $40 \%$ size reduction can be realized in the new structure. The new structure also provides flexibilities in introducing additional functionalities to the bandstop resonators. By embedding varactors in the slotted ground structure, tunable bandstop resonators can be implemented and $13 \%$ tuning range centered at 2.36 or 2.67 GHz have been obtained.
\end{abstract}

Index Terms-Defected ground structure, microstrip, slotted ground structure, tunable bandstop resonator.

\section{INTRODUCTION}

$\mathbf{T}$ RANSMISSION lines, when incorporated with patterned ground structures such as slots and holes, exhibit attractive characteristics including stopband and slow wave effect [1]-[3]. These patterned structures are sometimes referred to as "defected" ground structures or slotted ground structures [3]. When implemented in the ground plane, these structures enable the efficient usage of printed circuit boards (PCBs) in microwave hybrid modules and semiconductor substrates in monolithic microwave integrated circuits. Various slotted and defected ground structures have been proposed and applied for spurious-suppression of filters, harmonic tuning, and suppressions for amplifiers and antennas [4]-[10]. The dumbbell-shaped cell shown

Manuscript received February 28, 2007; revised June 9, 2007. This work was supported by the Specialized Research Fund for the Doctoral Program of Higher Education of China under Grant 20060614005, by the National Natural Science Foundation of China under Grant 90505001, by the University of Electronic Science and Technology of China under the Creative Research Team Program, and by the Hong Kong Research Grant Council under Grant CERG 611805.

X.-H. Wang is with the Institute of Applied Physics, University of Electronic Science and Technology of China, Chengdu 610054, China, and also with the Department of Electronic and Computer Engineering, Hong Kong University of Science and Technology, Kowloon, Hong Kong (e-mail: eexhwang@ust.hk).

B.-Z. Wang is with the Institute of Applied Physics, University of Electronic Science and Technology of China, Chengdu 610054, China.

H. Zhang was with the Department of Electronic and Computer Engineering, Hong Kong University of Science and Technology, Kowloon, Hong Kong. He is now with the Department of Electrical and Computer Engineering, University of Arizona, Tucson, AZ 85721 USA.

K. J. Chen is with the Department of Electronic and Computer Engineering, Hong Kong University of Science and Technology, Kowloon, Hong Kong (e-mail: eekjchen@ust.hk).

Color versions of one or more of the figures in this paper are available online at http://ieeexplore.ieee.org.

Digital Object Identifier 10.1109/TMTT.2007.904045 in Fig. 1(a) is one of the first defected ground structure proposed [2]. Based on this structure, many other modified and new patterned ground structures have been proposed to further improve the performance [11]-[14]. In [11] and [12], wide stopbands were obtained by cascading dumbbell-shaped resonant cells with different resonance frequencies in the ground plane. A superposition of an I- and $\mathrm{H}$-shaped defected resonant cell was proposed to design an ultra-wide stopband low-pass filter [13]. An H-shaped resonant cell was proposed, shown in Fig. 1(b), for its compact characteristics [14]. The circuit models for the patterned ground structures were also proposed [2], [15]. Recently, the conventional dumbbell-shaped resonant cell was modified by adding a patch in the middle of the defected holes [16], [17], as shown in Fig. 1(c). By adding a varactor between the ground and patch (where a bias can be applied with the help of external dc-bias networks), tunable bandstop resonators have been realized in the context of a coplanar waveguide (CPW). The merit of tunable resonators lies in the need of tunable amplifiers and antennas that are desired for software-defined radios or reconfigurable wireless systems. Tunable bandstop resonators can be used for the harmonic traps that can be integrated with tunable amplifiers or antennas to improve the circuit performance.

In this paper, we propose a microstrip bandstop resonator with a slotted ground structure [as shown in Fig. 1(d)] featuring compact size and wide spurious-free passband. Compared to the conventional dumbbell-, $\mathrm{H}$-, and modified dumbbell-shaped bandstop resonators, the proposed resonator cell exhibits $94 \%$, $57 \%$, and $88 \%$ size reduction, at a resonance frequency of $3.85 \mathrm{GHz}$. By introducing additional gaps in the slot, varactors can be embedded to the resonator cell and tunable bandstop resonators can be realized. The microstrip configuration also allows easy placement of the RF chokes that are required for the biasing circuits. The tunable bandstop resonator was demonstrated by implementing the proposed structure on a Rogers RT/Duroid 6010 PCB. A 13\% tuning range centered at 2.36 or $2.67 \mathrm{GHz}$ is achieved.

The design concept, compactness, and passband response of the proposed slotted ground structure are illustrated in Section II based on full-wave electromagnetic (EM) simulation. Tunable bandstop resonators are then discussed and verified by measurement results from a fabricated circuit in Section III. Section IV provides concluding remarks.

\section{Design AND Characteristics of the Proposed SLOTTED GROUND STRUCTURE}

\section{A. Compact Size}

For the typical dumbbell-shaped cell shown in Fig. 1(a), the resonance frequency $\left(f_{0}\right)$ depends on the physical dimensions 


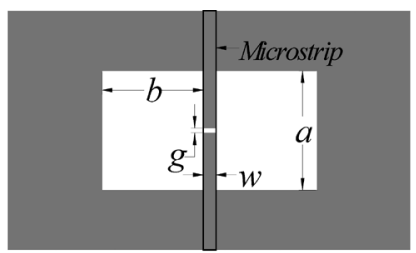

(a)

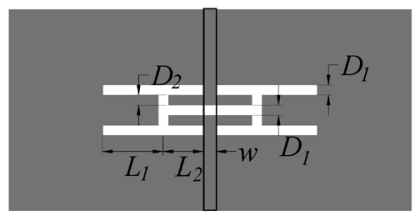

(b)

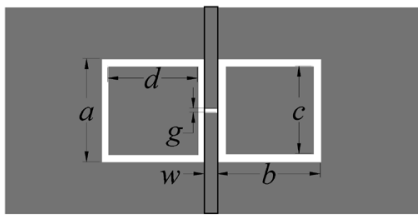

(c)

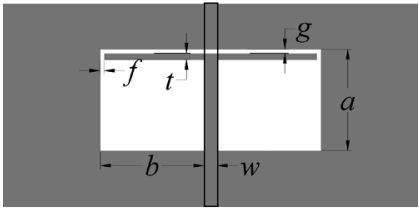

(d)

Fig. 1. Schematic layout of various slotted ground structures. (a) Dumbbellshaped defected ground structure (from [2]). (b) H-shaped defect ground structure (from [13]). (c) Modified tunable dumbbell-shaped defected ground structure (from [15]). (d) Our proposed slotted ground structure. The microstrip lines are outlined by the dark lines.

of the cell. For example, $f_{0}$ can be reduced by using smaller gap $g$, larger $a$ and $b$, or larger distance $w$ between the two bells. Since $g$ is generally limited by PCB fabrication techniques, increasing the size of the cell is the practical approach of reducing the resonance frequency. If there are floating patches inside the dumbbell-shaped cell, as shown in Fig. 1(c), $f_{0}$ will exhibit a shift toward lower values [16]. In the H-shaped cell [see Fig. 1(b)], $f_{0}$ is affected by the length of $L_{1}$ and $L_{2}$ and the width of $D_{1}$ and $D_{2}$. Once again, the lower $f_{0}$ is, the larger the physical dimensions. The proposed cell, as shown in Fig. 1(d), is based on moving the coupling gap to the edges of the slotted holes and lengthening the coupling slot. To compare the compactness of the four structures in Fig. 1, we simulated the transmission coefficient $\left(S_{21}\right)$ of microstrip lines on top of these structures. The simulations are performed using a full-wave EM simulator, i.e., Ansoft's High Frequency Structure Simulator (HFSS). The substrate used in the simulation has the same parameters as the Rogers RT/Duroid 6010 with a board thickness of $0.635 \mathrm{~mm}$, a dielectric constant of $\varepsilon_{r}=10.2$, and a loss tangent of 0.0023 . In the simulations, the width of the center metal traces in all the structures $(w)$ is kept at a fixed value and is the same as the width of the microstrip lines.

Fig. 2 shows the results of $S_{21}$ when the rectangular occupying areas of the four structures (as drawn in Fig. 1) are all the same, where $a=b=5 \mathrm{~mm}, c=d=4 \mathrm{~mm}, g=0.2 \mathrm{~mm}$,

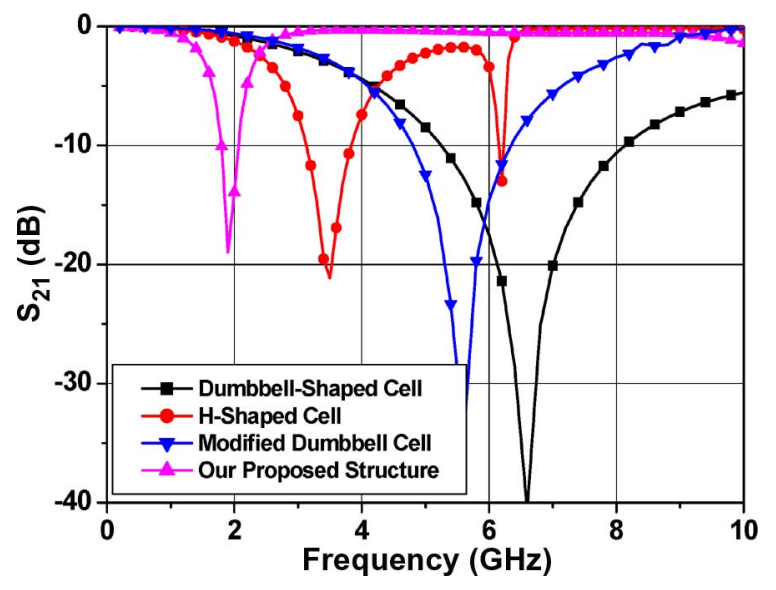

Fig. 2. Simulated results of the four structures (as drawn in Fig. 1) with the same occupying area.

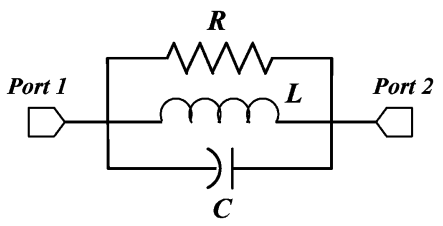

Fig. 3. Equivalent-circuit model of the bandstop resonators.

$t=0.3 \mathrm{~mm}, D_{1}=0.5 \mathrm{~mm}, D_{2}=1.75 \mathrm{~mm}, L_{1}=2 \mathrm{~mm}$, $L_{2}=3 \mathrm{~mm}$, and $w=0.635 \mathrm{~mm}$. $f_{0}$ of the proposed cell is $1.9 \mathrm{GHz}$, compared to $3.5 \mathrm{GHz}$ for the $\mathrm{H}$-shaped cell, 5.6 GHz for the modified dumbbell cell, and $6.6 \mathrm{GHz}$ for the conventional dumbbell cell.

The compactness of the proposed resonant cell can be understood based on the parameters extracted from the equivalent-circuit model (Fig. 3) proposed by Woo et al. [18]. Table I compares the extracted equivalent-circuit parameters of the proposed cell and the conventional dumbbell-shaped cell. It is observed that the capacitance of the proposed cell is approximately ten times that of the dumbbell-shaped cell, while there is little difference in the inductances. Thus, we can conclude that the extended gap length results in increased capacitance, and the increased capacitance leads to $f_{0}$ reduction and size reduction.

\section{B. Suppression of Spurious Response in a Wide Passband}

The proposed structure exhibits high- $Q$ resonance and spurious-free wide passband. With a fixed bandstop resonance frequency of $3.85 \mathrm{GHz}$, the physical parameters of the four bandstop resonators under study were designed and the simulated transmission coefficients are plotted in Fig. 4. Table II summarizes the physical dimensions of the four bandstop resonators. The proposed structure, once again, shows significant size reduction. The rectangular occupying area of the proposed cell is approximately $6 \%, 43 \%$, and $12 \%$ of the conventional dumbbell-, H-, and modified dumbbell-shaped cell, respectively.

The unloaded $Q$ factor $\left(Q_{u}\right)$ is an important parameter used to evaluate the intrinsic loss of a resonant circuit. From the equivalent-circuit parameters $R, L$, and $C$ of the four cells listed in Table II, their $Q_{u}$ 's are calculated by $Q_{u}=\omega_{0} R C$, where 
TABLE I

EXTRACTED EQUiVALENT-CircuIT PARAMETERS FOR THE Two STRUCTURES

\begin{tabular}{ccc}
\hline \hline & $\begin{array}{c}\text { Our proposed } \\
\text { structure }\end{array}$ & $\begin{array}{c}\text { Dumbbell-Shaped } \\
\text { defected ground } \\
\text { structure }\end{array}$ \\
\hline Resistance $(\mathrm{Ohm})$ & 2462.9 & 1434.2 \\
Inductance $(\mathrm{nH})$ & 3.5660 & 3.3019 \\
Capacitance $(\mathrm{pF})$ & 1.8681 & 0.1761 \\
Cutoff Frequency $(\mathrm{GHz})$ & 1.57 & 3.48 \\
Attenuation Pole & 1.95 & 6.60 \\
Location $(\mathrm{GHz})$ & \\
\hline \hline
\end{tabular}

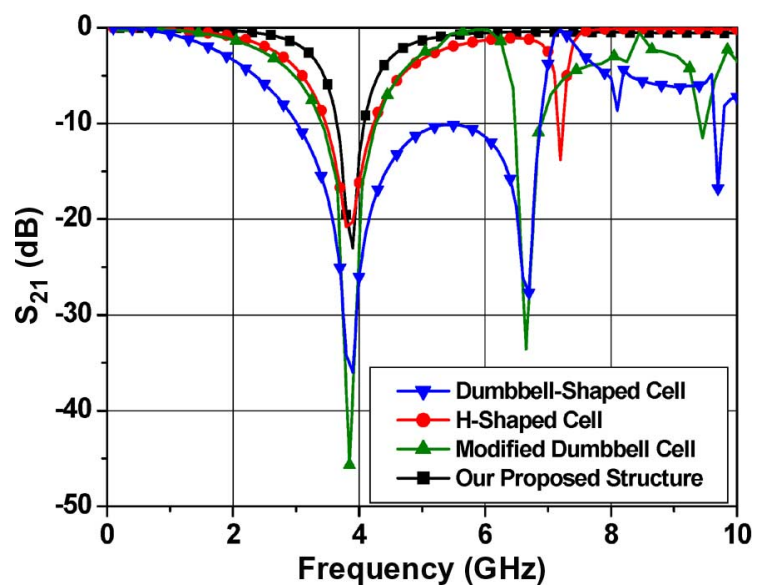

Fig. 4. HFSS simulation results of four bandstop resonators shown in Fig. 1.

TABLE II

SIZE AND $Q$ FACTOR COMPARISON OF FOUR RESONANT CELLS

\begin{tabular}{cccc}
\hline \hline & $\begin{array}{c}\text { Dimension Details } \\
(\text { Unit: } m \text { m })\end{array}$ & $\begin{array}{c}\text { Rectangular } \\
\text { Occupying } \\
\text { Area } \\
\left(\text { Unit: } m^{2}\right)\end{array}$ & $\begin{array}{c}\text { Unloade } \\
\mathrm{d}\end{array}$ \\
Q-Factor \\
\hline $\begin{array}{c}\text { Dumbbell-Shape } \\
\text { d Cell [2] }\end{array}$ & $\begin{array}{c}a=b=11.8, g=0.2, \\
w=0.635 .\end{array}$ & 285.973 & 10.9 \\
$\begin{array}{c}\text { H-Shaped Cell } \\
\text { [14] }\end{array}$ & $\begin{array}{c}D_{I}=0.5, D_{2}=1.1, L_{I}=2, \\
L_{2}=3, w=0.635 .\end{array}$ & 39.3495 & 11.1 \\
$\begin{array}{c}\text { Modified } \\
\text { Dumbbell-Shape } \\
\text { d Cell [16] }\end{array}$ & $\begin{array}{c}a=b=8.4, c=d=7.4, \\
g=0.2, w=0.635 .\end{array}$ & 146.454 & 17.1 \\
$\begin{array}{c}\text { Our proposed } \\
\text { slotted ground } \\
\text { structure }\end{array}$ & $\begin{array}{c}a=3, b=2.5, f=0.2, \\
g=0.2, t=0.3, w=0.635 .\end{array}$ & 16.905 & 51.2 \\
\hline \hline
\end{tabular}

$\omega_{0}$ is the resonant angularf frequency. It is 51.2 for our proposed structure, 11.1 for the $\mathrm{H}$-shaped resonant cell, 10.9 for the traditional dumbbell cell. and 17.1 for the modified dumbbell cell. Our proposed resonator structure has the highest unloaded $Q$ factor, reflecting its low intrinsic loss. In addition, the spurious response observed between the fundamental resonance frequency and $10 \mathrm{GHz}$ in three previous proposed structures does not occur in the newly proposed slotted ground structure, a favorable feature for bandstop filters. This phenomenon can be understood by considering the proposed structure as a stepped-impedance (achieved by the narrow slot on the top and wide slot at the bottom) slotline resonator, which is capable of shifting the spurious harmonic responses to higher frequencies [19].

For applications that require narrowband rejections, high- $Q$ factor is preferred for the bandstop resonator. Recently, it was

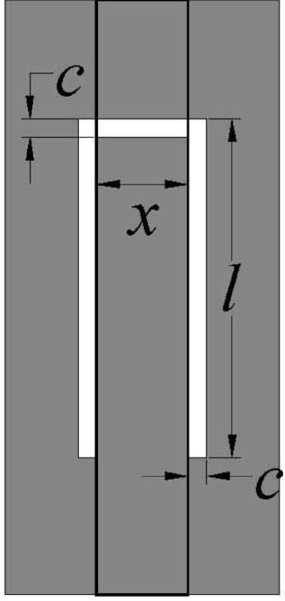

(a)

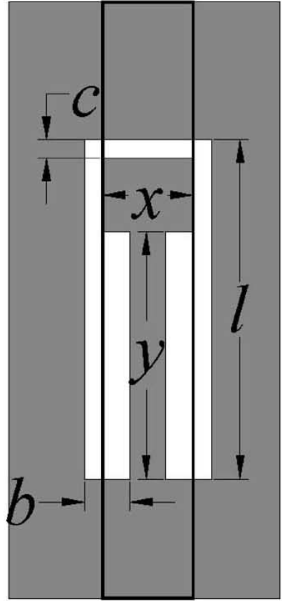

(b)

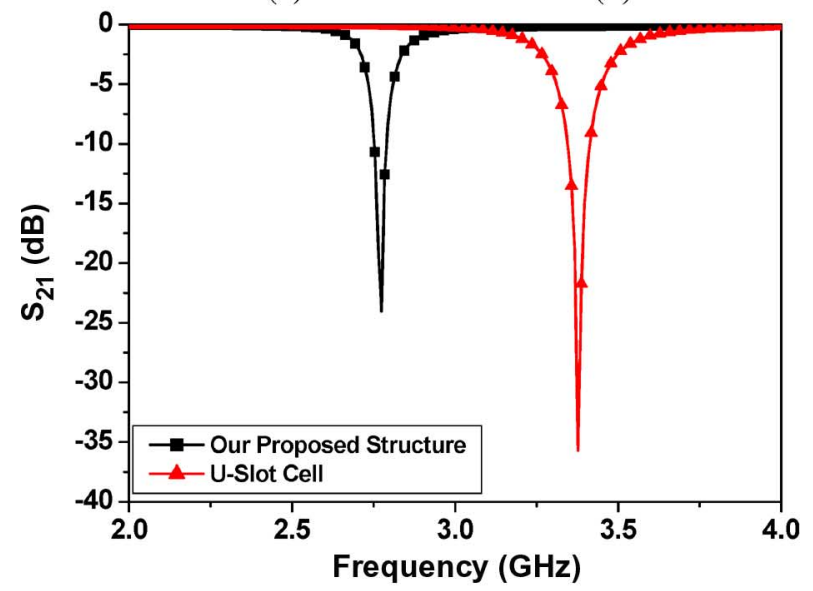

(c)

Fig. 5. Layout of two cells and their simulation results. (a) U-slot cell. (b) Our proposed cell. (c) Simulated $S_{21}$. (Where $b=0.6 \mathrm{~mm}, c=0.2 \mathrm{~mm}, l=9 \mathrm{~mm}$, $x=1 \mathrm{~mm}$, and $y=7 \mathrm{~mm}$.)

shown [18] that the loaded $Q$ factor of a U-slot structure [as drawn in Fig. 5(a)] increases when the distance $(x)$ between the two slots in the U-shape decreases. The same trend of the loaded $Q$ factor has also been observed in our proposed structure [as drawn in Fig. 5(b)]. On the other hand, our proposed structure offers more compact size compared to the U-slot structure. A comparison between the two structures (ours and the U-slot) is illustrated in Fig. 5(c). With the same occupying area and the same slot width, our proposed structure possesses similar high $Q_{u}$ (610.7 compared to 648.5), but exhibits lower resonant frequency.

\section{TUNABLE BANDSTOP RESONATORS}

\section{A. Slotted Ground Structure for Tunable Bandstop Resonator}

In Section II, we proposed a new bandstop resonator cell, which features a longer slot compared to the conventional dumbbell-shaped cell. This slot increases the effective capacitance of the $L C$ resonant tank significantly, leading to size reduction. In addition, the long ground lines along the slot provide flexibilities in modifying the structure for enhanced functionalities. In Fig. 6, the original and a modified structure 


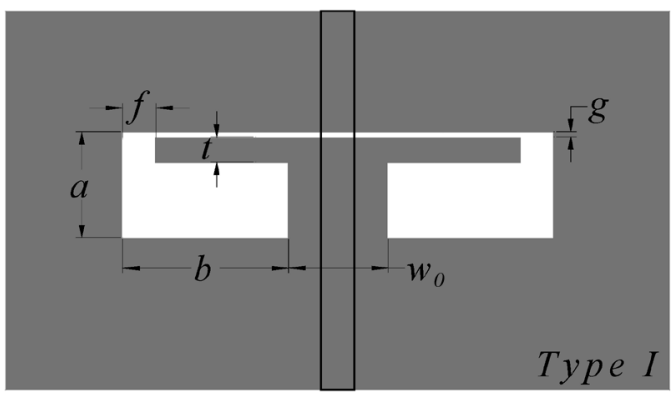

(a)

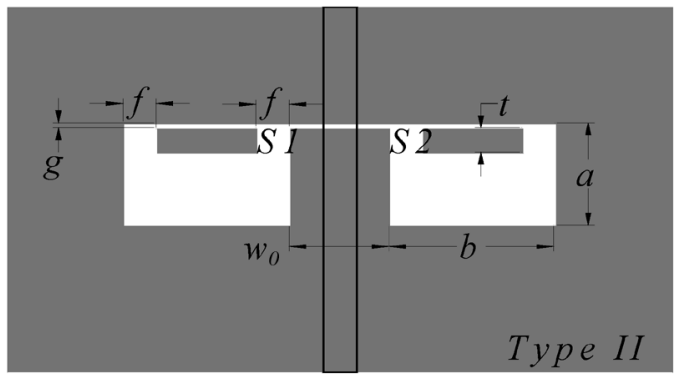

(b)

Fig. 6. Layout of two types of slotted ground structure.

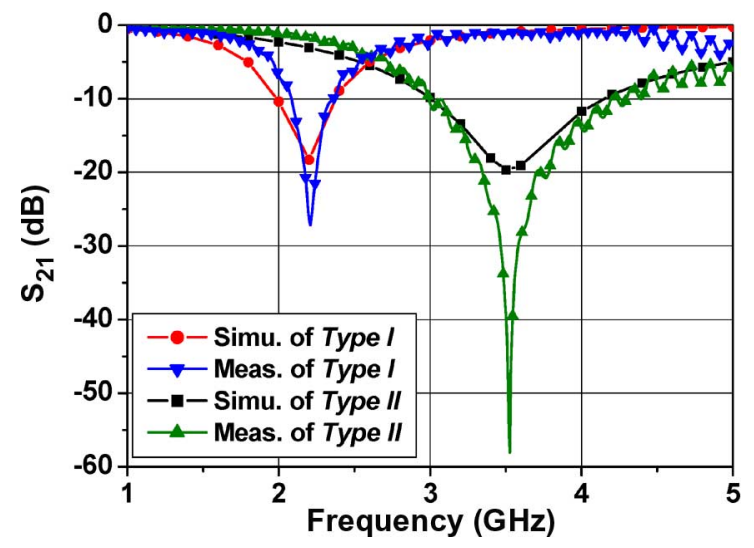

Fig. 7 Simulated and measured results of Type $I$ and $I I$ slotted ground structures shown in Fig. 6.

TABLE III

EXTRACTED EQUIVALENT-CIRCUIT PARAMETERS FOR Type I AND II

\begin{tabular}{ccc}
\hline \hline & Type I & Type II \\
\hline Inductance $(\mathrm{nH})$ & 3.8602 & 3.8801 \\
Capacitance (pF) & 1.3558 & 0.5269 \\
Cutoff Frequency (GHz) & 1.69 & 2.32 \\
Attenuation Pole & 2.20 & 3.52 \\
Location (GHz) & \\
\hline \hline
\end{tabular}

are shown. Type $I$ is our originally proposed cell discussed in Section II. Type II, shown in Fig. 6(b), is a modified Type I in which the ground lines along the slot are disconnected from and capacitive coupled to the center ground line. The strength of the coupling is determined by the size of the slots $S 1$ and $S 2$, shown in Fig. 6(b). We found that the value of the coupling capacitance determines the resonance frequency of the proposed cell. The two cells in Fig. 6 were designed and fabricated with

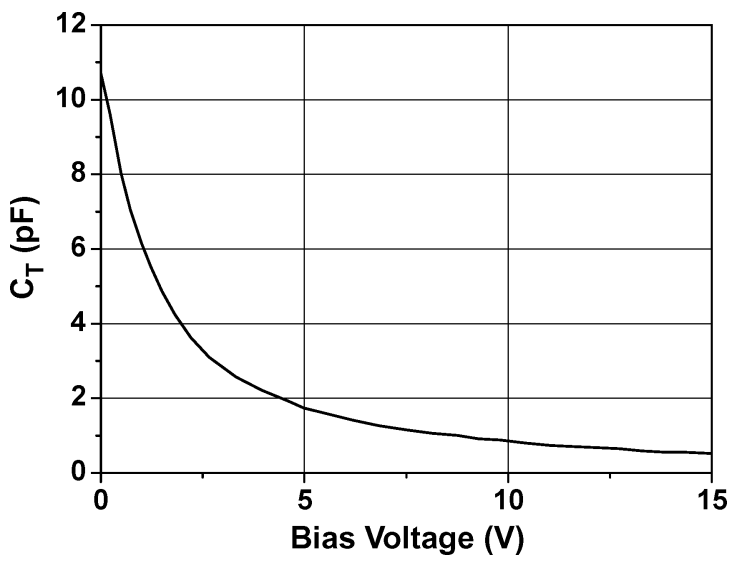

Fig. 8. $C-V$ characteristics of the varactor used in the tunable bandstop resonators.

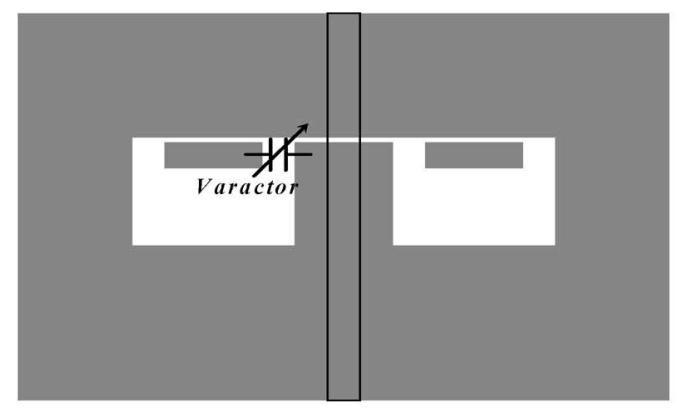

(a)

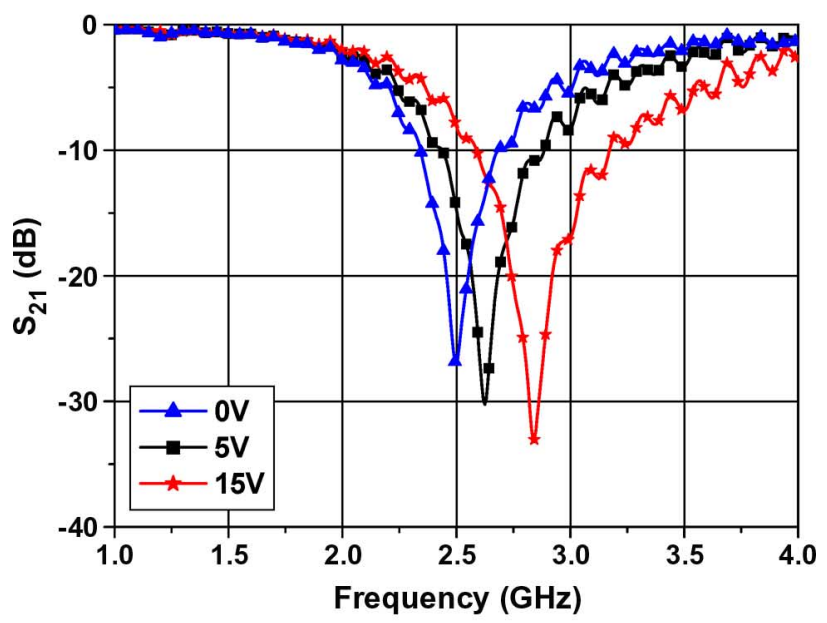

(b)

Fig. 9. Tunable bandstop resonator with one varactor. (a) Schematic layout. (b) Measured results with three different tuning biasing voltages.

the following physical dimensions: $a=4.2 \mathrm{~mm}, b=5 \mathrm{~mm}$, $f=1 \mathrm{~mm}, g=0.2 \mathrm{~mm}, t=1 \mathrm{~mm}$, and $w_{0}=3 \mathrm{~mm}$.

The simulated and measured results of Type $I$ and $I I$ cells are plotted in Fig. 7. The resonance frequency is 2.20 and $3.52 \mathrm{GHz}$ for the Type I and $I I$ cells, respectively. Table III summarizes the equivalent-circuit parameters of the two slotted ground structure resonators. It can be observed that the effective capacitance in the Type II resonator is reduced, leading to a higher resonance frequency. Thus, if the coupling capacitance between the arms and center ground line can be electronically controlled, 


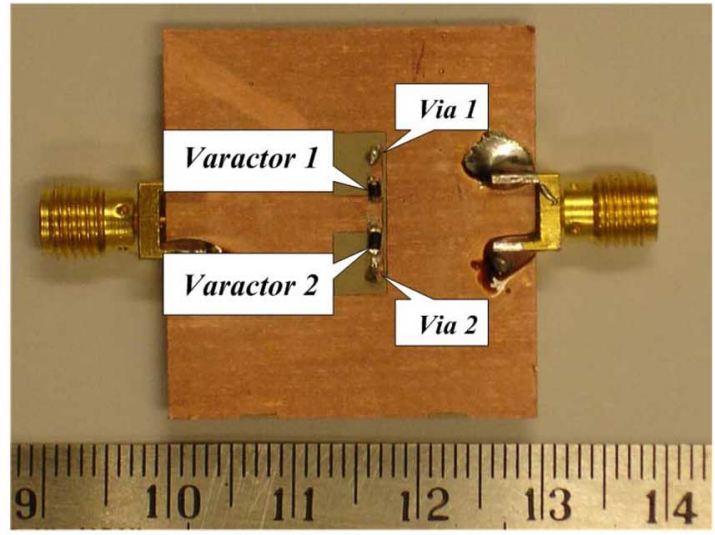

(a)

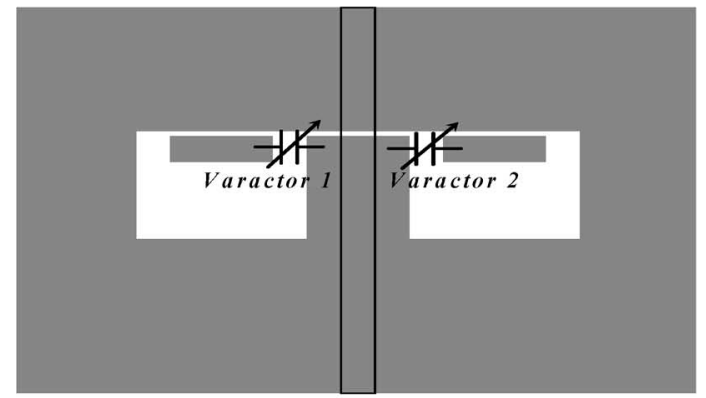

(b)

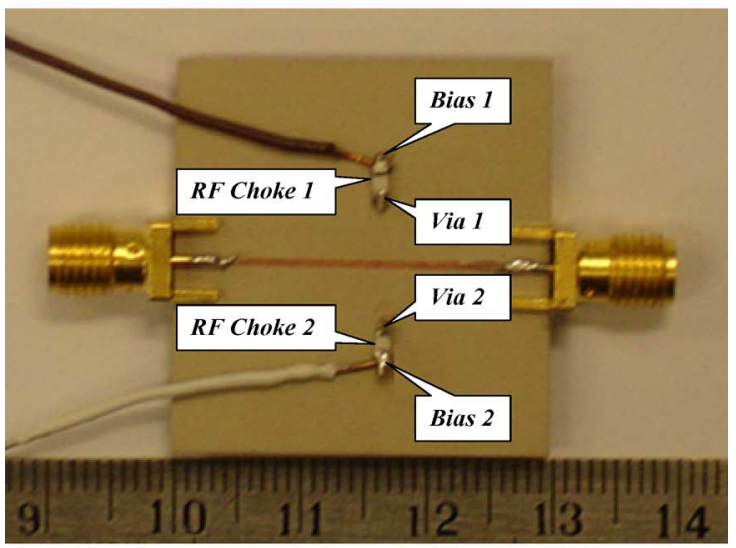

(c)

Fig. 10. Tunable bandstop resonator. (a) View of the ground plane. (b) Schematic drawing of the ground plane. (c) View of the signal plane.

a bandstop resonator with tunable resonance frequency can be achieved. Since Type I represents the situation when the slot ( $S 1$ and $S 2$ ) coupling capacitance is infinite, the resonance frequency of Type I represents the theoretical lower limit for the tuning range. Type II represents the case when the slot coupling capacitance is negligibly small, setting up the theoretical upper limit for the tuning range. The tuning of the slot capacitance can be achieved by inserting varactors into the slots while applying dc bias at the open ends of the arms.

\section{B. Experimental Results of the Tunable Bandstop Resonator}

In our study, the MV31020-46 varactors from the MDT Corporation, Westford, MA, are used in the $S 1$ and $S 2$ slots in

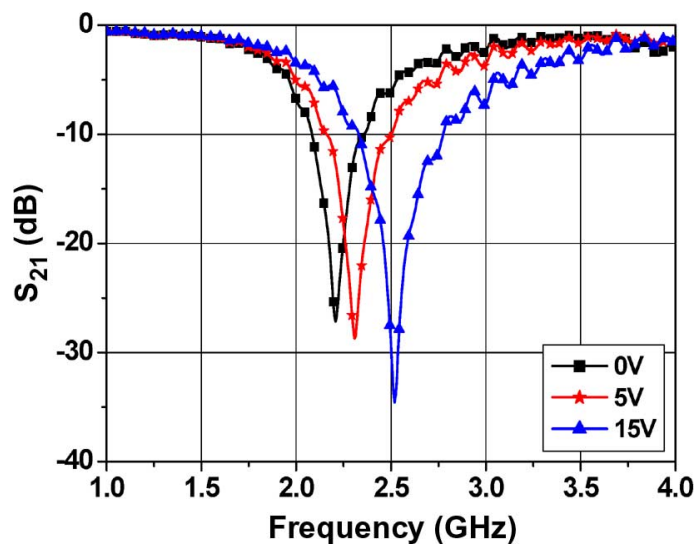

Fig. 11. Measured results of the tunable bandstop resonator with two varactors under three different biasing voltages.

Fig. 6(b). Fig. 8 shows the capacitance-voltage $(C-V)$ characteristics of this varactor. The tuning range of capacitance is from 0.5 to $10.5 \mathrm{pF}$. When one slot is open and one slot is occupied by one varactor, as shown in Fig. 9(a), 12.8\% tuning range centered at $2.67 \mathrm{GHz}$ is achieved in measurement, as shown in Fig. 9(b).

We can also apply two varactors in both of slots $S 1$ and $S 2$, as shown in Fig. 10. DC bias needs to be provided and RF chokes are needed between the dc voltage source and the RF current path. The placement of the dc bias and the RF chokes should have minimum impact on the resonant cell in the ground plane, thus making it difficult to be implemented in the ground plane. The microstrip configuration allows the placement of the RF chokes on the signal plane, as shown in Fig. 10(c). With the two varactors simultaneously controlled by a single biasing voltage, the measured results are shown in Fig. 11. A 13.2\% tuning range centered at $2.35 \mathrm{GHz}$ was obtained.

The simulated and measured results are plotted together in Fig. 12 when the capacitance of the varactors are at the available minimum and maximum values, together with a simulated curve when the varactor's capacitance was set at a unrealistic small value of $0.01 \mathrm{pF}$. The challenge in realizing large tuning range lies in obtaining varactors that can be tuned to very small capacitance value. From Fig. 12, it can be seen that there is a good agreement when $C=10.5 \mathrm{pF}$ and small difference when $C=0.5 \mathrm{pF}$ between the measured and simulated resonance frequency. The difference at a lower capacitance value is a result of the parasitics of the varactor. Due to the parasitic inductance $\left(L_{p}\right)$ and capacitance $\left(C_{p}\right)$ of varactors, there is a $0.11-\mathrm{GHz}$ difference in $f_{0}$ between the simulation and measurement results. The effect of the parasitics is especially significant when the varactor is tuned to small capacitance value. Fig. 13 shows the equivalent-circuit model of the varactor. It is obvious that $L_{p}$ and $C_{p}$ would affect the effective inductance and capacitance of the varactor. By adding $L_{p}$ and $C_{p}$ in the HFSS simulation, the phenomena has been validated. Since the capacitance of the varactor cannot reach zero, it cannot work as an open component absolutely, limiting the tuning range at the upper end. The simulated result by HFSS by assuming a $C=0.01 \mathrm{pF}$ is plotted in Fig. 12. It is found that $f_{0}$ is close to $3.5 \mathrm{GHz}$ (the resonance frequency of the Type II cell in Fig. 6) if a varactor with a 0.01-pF minimum capacitance is available. 


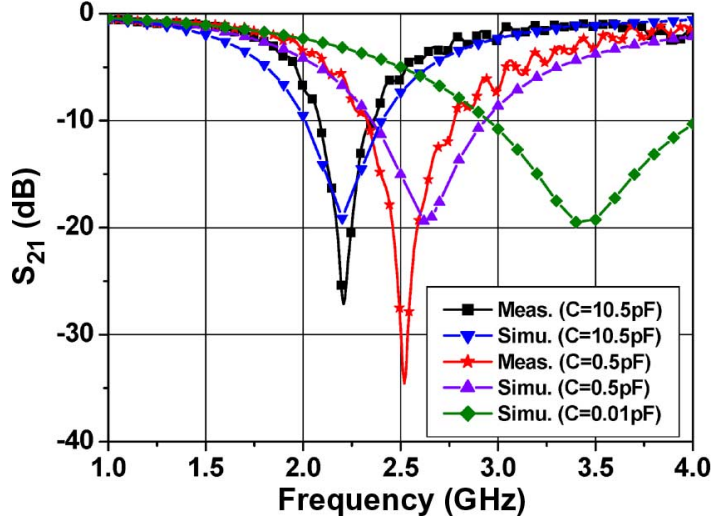

Fig. 12. Measured and simulated results of a tunable bandstop resonator with two varactors.

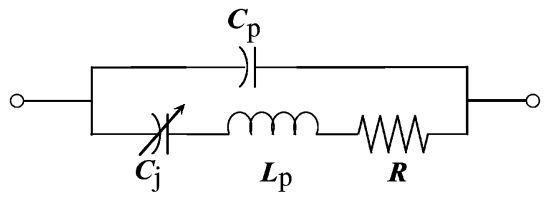

Fig. 13 Equivalent-circuit model of the varactor. Parasitic capacitance and inductance are represented by $C_{p}$ and $L_{p}$, respectively.

\section{CONCLUSION}

We have proposed a new slotted ground bandstop resonator featuring a lengthened coupling slot that results in increased capacitance and reduced resonance frequency. Compared to other previously proposed bandstop resonator structures in the ground plane, the physical size of the new resonator is greatly reduced with the same resonance frequency. The spurious response is pushed to much higher frequencies, resulting in a spurious-free wide passband. The proposed structure also allows us to design tunable or reconfigurable bandstop resonators. By embedding varactors in the proposed structure, a tuning range of $\sim 13 \%$ centered at 2.36 and $2.67 \mathrm{GHz}$ was achieved. Good agreements are achieved between simulations and measurements. The tunable bandstop resonator can be integrated with tunable amplifiers or antennas for enhanced performance.

\section{ACKNOWLEDGMENT}

The authors would like to thank K. W. Chan, Hong Kong University of Science and Technology (HKUST), Kowloon, Hong Kong, for his valuable help with fabrication and testing.

\section{REFERENCES}

[1] V. Radisic, Y. Qian, R. Coccioli, and T. Itoh, "Novel 2-D photonic bandgap structure for microstrip lines," IEEE Microw. Guided Wave Lett., vol. 8, no. 2, pp. 69-71, Feb. 1998.

[2] D. Ahn, J.-S. Park, C.-S. Kim, J. Kim, Y. Qian, and T. Itoh, “A design of the low-pass filter using the novel microstrip defected ground structure," IEEE Trans. Microw. Theory Tech., vol. 49, no. 1, pp. 86-93, Jan. 2001.
[3] C. Caloz, H. Okabe, T. Iwai, and T. Itoh, "A simple and accurate model for microstrip structures with slotted ground plane," IEEE Microw. Wireless Compon. Lett., vol. 14, no. 4, pp. 133-135, Apr. 2004.

[4] J.-S. Lim, H.-S. Kim, J.-S. Park, D. Ahn, and S. Nam, "A power amplifier with efficiency improved using defected ground structure," IEEE Microw. Wireless Compon. Lett., vol. 11, no. 4, pp. 170-172, Apr. 2001.

[5] J.-S. Lim, C.-S. Kim, J.-S. Park, D. Ahn, and S. Nam, "Design of 10 $\mathrm{dB} 90$ " branch line coupler using microstrip line with defected ground structure," Electron. Lett., vol. 36, no. 21, pp. 1784-1785, Oct. 2000.

[6] J.-S. Lim, S.-W. Lee, C.-S. Kim, J.-S. Park, D. Ahn, and S. Nam, "A 4:1 unequal Wilkinson power divider," IEEE Microw. Wireless Compon. Lett., vol. 11, no. 3, pp. 124-126, Mar. 2001.

[7] Y. J. Sung and Y.-S. Kim, "An improved design of microstrip patch antennas using photonic bandgap structure," IEEE Trans. Antennas Propag., vol. 53, no. 5, pp. 1799-1804, May 2005.

[8] H. Liu, Z. Li, X. Sun, and J. Mao, "Harmonic suppression with photonic bandgap and defected ground structure for a microstrip patch antenna," IEEE Microw. Wireless Compon. Lett., vol. 15, no. 2, pp. 55-56, Feb. 2005.

[9] Y. Chung, S.-S. Jeon, S. Kim, D. Ahn, J.-I. Choi, and T. Itoh, "Multifunctional microstrip transmission lines integrated with defected ground structure for RF front-end application," IEEE Trans. Microw. Theory Tech., vol. 52, no. 5, pp. 1425-1432, May 2004.

[10] J.-S. Lim, C.-S. Kim, Y.-T. Lee, D. Ahn, and S. Nam, "A spiral-shaped defected ground structure for coplanar waveguide," IEEE Microw. Wireless Compon. Lett., vol. 12, no. 9, pp. 330-332, Sep. 2002.

[11] H.-W. Liu, Z.-F. Li, X.-W. Sun, and J.-F. Mao, "An improved 1-D periodic defected ground structure for microstrip line," IEEE Microw. Wireless Compon. Lett., vol. 14, no. 4, pp. 180-182, Apr. 2004.

[12] J.-S. Park, J.-S. Yun, and D. Ahn, "A design of the novel coupled-line bandpass filter using defected ground structure with wide stopband performance," IEEE Trans. Microw. Theory Tech., vol. 50, no. 9, pp. 2037-2043, Sep. 2002.

[13] H.-J. Chen, T.-H. Huang, C.-S. Chang, L.-S. Chen, N.-F. Wang, Y.-H. Wang, and M.-P. Houng, "A novel cross-shape DGS applied to design ultra-wide stopband low-pass filters," IEEE Microw. Wireless Compon. Lett., vol. 16, no. 5, pp. 252-254, May 2006.

[14] M. K. Mandal and S. Sanyal, "A novel defected ground structure for planar circuits," IEEE Microw. Wireless Compon. Lett., vol. 16, no. 2, pp. 93-95, Feb. 2006.

[15] J.-S. Hong and B. M. Karyamapudi, "A general circuit model for defected ground structures in planar transmission lines," IEEE Microw. Wireless Compon. Lett., vol. 15, no. 10, pp. 706-708, Oct. 2005.

[16] A. M. E. Safwat, F. Podevin, P. Ferrari, and A. Vilcot, "Tunable bandstop defected ground structure resonator using reconfigurable dumbbell-shaped coplanar waveguide," IEEE Trans. Microw. Theory Tech., vol. 54, no. 9, pp. 3559-3564, Sep. 2006.

[17] C. C. Wong and C. E. Free, "DGS pattern with enhanced effective capacitance," Electron. Lett., vol. 42, no. 8, pp. 470-471, Apr. 2006.

[18] D. J. Woo, T. K. Lee, J. W. Lee, C. S. Pyo, and W. K. Choi, "Novel U-slot and V-slot DGSs for bandstop filter with improved $Q$ factor," IEEE Trans. Microw. Theory Tech., vol. 54, no. 6, pp. 2840-2847, Jun. 2006.

[19] M. Makimoto and S. Yamashita, Microwave Resonators and Filters for Wireless Communications-Theory and Design. Berlin, Germany: Springer-Verlag, 2001.

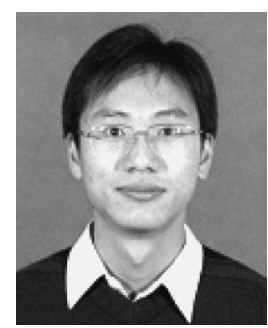

Xiao-Hua Wang was born in Jiangsu Province, China, in 1980. He received the B.E. degree in electromagnetic field and microwave technology and M.S. degree in radio physics from the University of Electronic Science and Technology of China (UESTC), Chengdu, China, in 2002 and 2005, respectively, and is currently working toward the Ph.D. degree in radio physics at UESTC.

From November 2006 to May 2007, he was a Research Assistant with the Department of Electronic and Computer Engineering, Hong Kong University of Science and Technology. His research interests include computational electromagnetics, transmission line theory, and microwave passive components. 


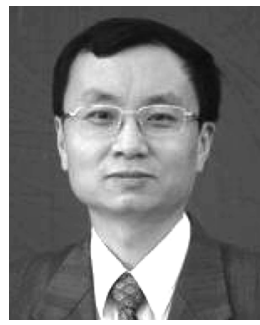

Bing-Zhong Wang (M'06) received the Ph.D. degree in electrical engineering from the University of Electronic Science and Technology of China (UESTC), Chengdu, China, in 1988.

In 1984, he joined UESTC, where he is currently a Professor. He has been a Visiting Scholar with the University of Wisconsin-Milwaukee, a Research Fellow with the City University of Hong Kong, and a Visiting Professor with the Electromagnetic Communication Laboratory, Pennsylvania State University, University Park. His current research interests are in the areas of computational electromagnetics, antenna theory and techniques, electromagnetic compatibility analysis, and computer-aided design for passive microwave integrated circuits.

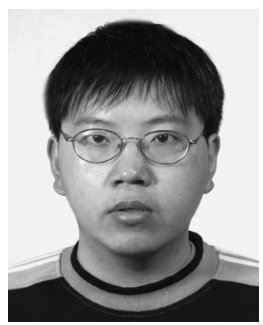

Hualiang Zhang was born in Wuhan, Hubei Province, China. He received the B. Eng. degree in electrical engineering and information science from the University of Science and Technology of China (USTC), Hefei, China, in 2003, and the Ph.D. degree in electronic and computer engineering from the Hong Kong University of Science and Technology (HKUST), Kowloon, Hong Kong, in 2007.

In May, 2007, he joined the Department of Electrical and Computer Engineering, University of Arizona, Tucson, where he is currently a Post-Doctoral Fellow. His research interests include design and synthesis of microwave filters, microelectromechanical systems (MEMS) technologies (especially their applications to RF passive components), and optimization techniques.

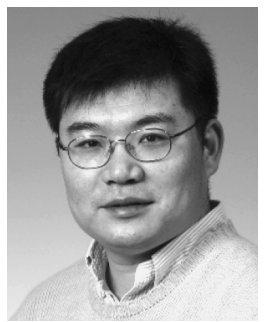

Kevin J. Chen (M'96-SM'06) received the B.S. degree in electronics from Peking University, Beijing, China, in 1988, and the Ph.D. degree from the University of Maryland at College Park, in 1993.

From January 1994 to December 1995, he was a Research Fellow with National Telephone and Telegraph (NTT) Large Signal Integration (LSI) Laboratories, Atsugi, Japan, where he was engaged in research and development of functional quantum effect devices and heterojunction field-effect transistors (HFETs). In particular, he developed device technologies for monolithic integration of resonant tunneling diodes and HFETs on both GaAs and InP substrates for applications in ultrahigh-speed signal processing and communication systems. He also developed the Pt-based buried gate technology that is widely used in enhancement-mode HEMT and pseudomorphic HEMT (pHEMT) devices. From 1996 to 1998, he was an Assistant Professor with the Department of Electronic Engineering, City University of Hong Kong, where he performed research on high-speed device and circuit simulations. In 1999, he joined the Wireless Semiconductor Division, Agilent Technologies, Santa Clara, CA, where he was involved with enhancement-mode pHEMT RF power amplifiers used in dual-band global system for mobile communication (GSM)/digital communication system (DCS) wireless handsets. His research with Agilent Technologies has covered RF characterization and modeling of microwave transistors, RF integrated circuits (ICs), and package design. In November 2000, he joined the Department of Electronic and Computer Engineering, Hong Kong University of Science and Technology (HKUST), Kowloon, Hong Kong, as an Assistant Professor and, in 2006, became an Associate Professor. He has authored or coauthored over 150 publications in international journals and conference proceedings. With HKUST, his group has performed research on high-speed compound semiconductor devices and ICs, novel III-nitride device technologies, III-nitride and silicon-based microelectromechanical systems (MEMS), silicon-based RF/microwave passive components, RF packing technology, and microwave filter design. 